\title{
CAPABILITY OF OPTICAL CODE-BASED MPLS (OC-MPLS)
}

\author{
Ken-ichi Kitayama ${ }^{1}$, Kiyoshi Onohara ${ }^{1}$ and Masayuki Murata ${ }^{2}$ \\ ${ }^{1}$ Osaka University Department of Electronics and Information Systems, 2-1 Yamadaoka, \\ Suita,Osaka,565-0871,Japan \\ kitayama@comm.eng.osaka-u.ac.jp, onohara@comf5.comm.eng.osaka-u.ac.jp \\ ${ }^{2}$ Osaka University Cybermedia Center, 2-1 Yamadaoka, Suita, Osaka, 565-0871, Japan \\ muratalics.es.osaka-u.ac.jp
}

Abstract: We will focus on the novel applications of optical code-base MPLS, so-called OC-MPLS to demonstrate its versatile capability. The $\mathrm{OC}$-photonic label can be used as an identifier of the packet or flow encapsulated in an optical frame. The OC-photonic label is an optical code consisting of a sequence of optical chip pulses, and its recognition is performed based upon ultrafast time-domain optical correlation. The main application is to the label switched path (LSP) switching, but the other applications include $\mathbb{I}$ packet routing as well as flow classification for supporting QoS capabilities. In the optical implementations, two types of match algorithms of OC-photonic labels such as exact match for the LSP and flow classification as well as longest prefix match for IP packet routing are presented. Finally, the exact match, longest prefix match, and classification of optical packet flows will be experimentally demonstrated at the bit rate of $10 \mathrm{~Gb} / \mathrm{s}$.

Key words: MPLS, optical code, photonic label, $\mathbb{P}$ packet, optical path, flow, optical implementation

\section{INTRODUCTION}

Recent progress of wavelength division multiplexing (WDM) technology has significantly increased the point-to-point link capacity, providing scalable bandwidth for rapidly growing internet traffic demands. However, the capacity increase tends to shift the network capacity bottleneck from the link to the node due to rather slow packet processing capability in electronic layer at the node. 
One promising technique to alleviate the bottleneck is via circuitswitched MPL(ambda)S or MP $\lambda S$ (multi-protocol lambda switching) technology $[1,2]$. The MPLS performs packet forwarding decisions only at the edges by eliminating forwarding task at the core nodes, thus allowing the core nodes to perform only switching. MP $\lambda S$ is an extension of MPLS concept by provisioning wavelength path or optical path to establish a logical topology over WDM link network [3]. MP $\lambda S$ enables traffic engineering by providing optical paths where bandwidths or wavelengths are required. It allows the cut-through of the traffic not to be terminated at the node, and this is powerful to alleviate the load of router at the node. However, MP $\lambda S$ still poses several limitations due to the facts that [4];

The unit of path granularity is of wavelength capacity, which may be sometimes too large to accommodate the end-to-end traffic within the MPLS domain.

The number of available wavelengths is too small for the label space to accommodate all the paths required (Table 1) as the wavelength resource is not abundant.

Aggregation of packet flows having the same wavelength labels may not be feasible. The aggregation may require the label exchange within the network, but it needs the wavelength change at the node.

Table 1. Photonic label space[5]

\begin{tabular}{|c|c|c|c|}
\hline Code & Number & Label processing & Issues \\
\hline Wavelength & $\sim 1,000$ & $\begin{array}{c}\text { Simple } \\
\text { Optical filter }\end{array}$ & Flow control \\
\hline Subcarrier & $\sim 100$ & $\begin{array}{c}\text { Simple } \\
\text { RF filter }\end{array}$ & $<40 \mathrm{Gbit} / \mathrm{s}$ \\
\hline Optical code & Abundant & $\begin{array}{c}\text { Analog \& } \\
\text { relatively simple, } \\
\text { Passive waveguide }\end{array}$ & $\begin{array}{c}\text { Impairment due to } \\
\text { fiber dispersion }\end{array}$ \\
\hline
\end{tabular}

Optical packet switching might be an ultimate solution in the long run to overcome the above problems of MP $\lambda \mathrm{S}$. It has been extensively studied; for example, the European KEOPS project has shown a broadcast-and-select switch [6], where wavelength converters are used to perform space switching and delay selection. Packet contention at the output ports can be resolved by utilizing fiber delay lines, but a large number of semiconductor optical amplifier (SOA) gates are required for the broadcast-and-select stage. Another example is the WASPNET prototype [7] that uses currently available optical devices such as tunable wavelength converters, arrayed waveguide gratings (AWG), and fiber delay lines to resolve contention. However, these optical packet switches still rely on relatively slow electronic 
header processing and hence can provide the line interface only up to the bit rate of $10 \mathrm{~Gb} / \mathrm{s}$.

To overcome both the problems of circuit-switched MP $\lambda S$ and slow electronic header processing in optical packet switchings, optical codebased photonic label MPLS, so-called OC-MPLS has recently been proposed [4]. Here, OC-MPLS is referred to a special class of Generalized MPLS (GMPLS) which incorporates OC-photonic label as the generic label entry. OC-MPLS is a framework in which circuit-switched routing for stream and burst data as well as optical packet switching can be supported.

In this paper, we will focus on the novel applications of OC-MPLS to demonstrate its versatile capability. The OC-photonic label can be used as an identifier of the data encapsulated in an optical frame. The OC-photonic label is an optical code consisting of a sequence of optical chip pulses, and its recognition is performed based upon ultrafast time-domain optical correlation. The main application is to the label switched path (LSP) switching, and furthermore the other applications include IP packet routing as well as flow classification for implementing QoS capabilities. In the optical implementations, two types of match algorithms of OC-photonic labels such as exact match for the LSP and flow classification as well as longest prefix match for $\mathbb{P}$ packet routing are presented. Finally, the exact match, longest prefix match, and classification of optical packet flows will be experimentally demonstrated at the bit rate of $10 \mathrm{~Gb} / \mathrm{s}$.

\section{APPLICATIONS OF OC-PHOTONIC LABELS}

\section{$2.1 \quad$ OC-MPLS}

Before going into the applications, we will briefly review the concept of OC-MPLS [4]. The concept of OC-MPLS network is schematically illustrated in Fig.1. The label switching is distinct from conventional IP routing architecture in that MPLS uses just one forwarding algorithm of exact match on the label in the shim header. When the ingress photonic label switching router (PLSR) receives a packet without label from the IP router, the PLSR determines the forward equivalent class (FEC) of this packet and the next hop and attaches the OC-photonic label. The core PLSR carries out the label switching and swaps the label in optical layer. The egress PLSR strips the label from the packet and hands the packet to the IP router.

The PLSR may have functionality of either optical crossconnect switch or packet switch. As shown in Fig.2, a basic idea is to encapsulate into an optical frame the various types of data by attaching the $\mathrm{OC}$-photonic labels as the identifier, and then the identifier can be processed and recognized in 
optical layer by exploiting ultrafast OC-photonic label recognition, followed by controlling the tasks of either switching or flow classification based upon the information of the header. It should be noted that the IP packet routing using OC-photonic label is not on the main focus but the applications are not limited within the OC-MPLS but also extended conventional IP packet routing.

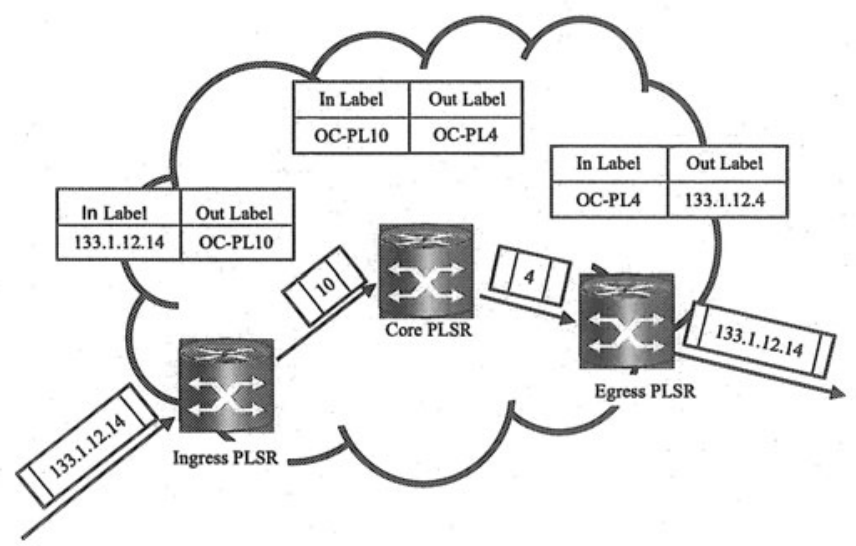

Figure 1. Concept of OC-MPLS

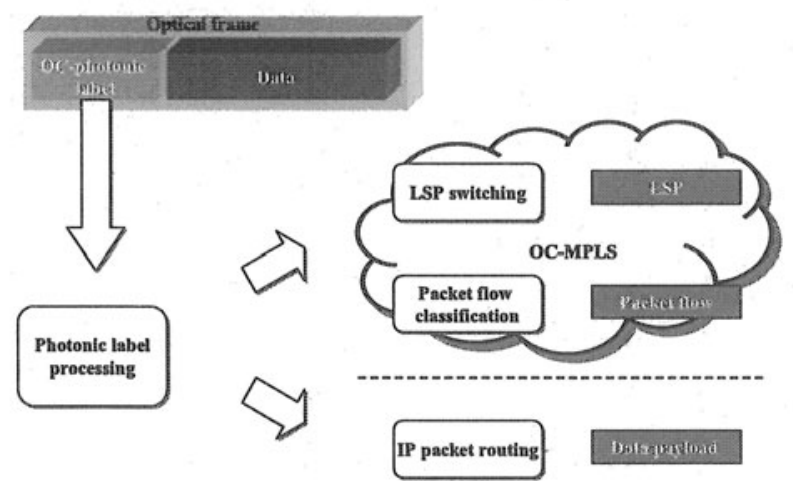

Figure 2. Basic idea using OC-photonic labels as the identifier of LSP, IP packet, and packet flow 


\subsection{IP routing : Longest prefix match}

In the conventional IP routing architecture, different functionality requires different forwarding algorithm. For example, forwarding of unicast packets requires longest match based upon the network layer destination address, forwarding of multicast packets requires longest match on the source network layer address plus the exact match on both source and destination network layer addresses, whereas unicast forwarding with Types of Services requires the longest match on the destination network layer address plus the exact match on the Types of Services bits carried in the network layer header [8]. OC-photonic label enables both the two match algorithms; longest prefix match and exact match, and therefore the applications are not limited within the OC-MPLS but also extended conventional IP packet routing.

The architecture of the photonic packet router which performs split of the flows as well as packet forwarding is shown in Fig.4 [9]. First, the variable time gate operates on the incoming label. This determines the match algorithm. It can gate out one of the labels, for example, the destination and/or source labels. It can also gate out a part of the label for the longest match. Then, the photonic processor recognizes the label and generates the control signal to drive the optical switch for forwarding or some other task. The architecture will be varied with the longest match and exact match. The photonic label can be processed based on the optical correlation between the codes. The optical correlation is performed by matched filtering in time domain [9]. This will be elaborated in Sect.3. 
Exact 楽atch algorithm for MPLS

\begin{tabular}{l|l|l}
$\begin{array}{l}\text { Input address } \\
133.243 .145 .66\end{array}$ & \multicolumn{1}{c|}{$\begin{array}{c}\text { Routing table } \\
\text { Data (IP address) }\end{array}$} \\
\hline Address & \multicolumn{1}{c}{$\begin{array}{l}\text { Hit address } \\
1066\end{array}$} \\
\hline $0000-0255$ & $133.1 .44 .0-133.1 .44 .255$ \\
$1000-1066$ & $133.243 .145 .0-133.243 .145 .66$ \\
$1067-1255$ & $133.243 .145 .67-133.243 .145 .255$ \\
\end{tabular}

Longest prefix match algorithm for IP routing)

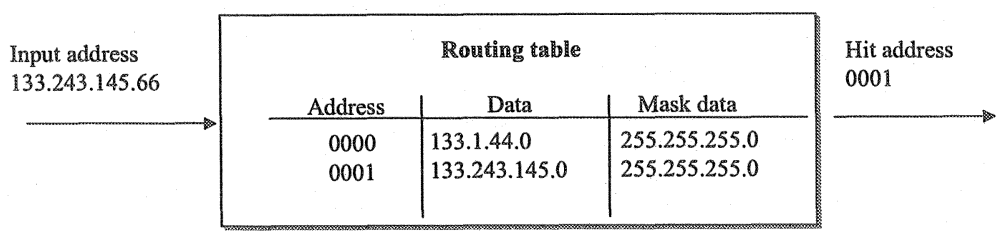

Figure 3. Forwarding algorithms : Longest prefix match and exact match

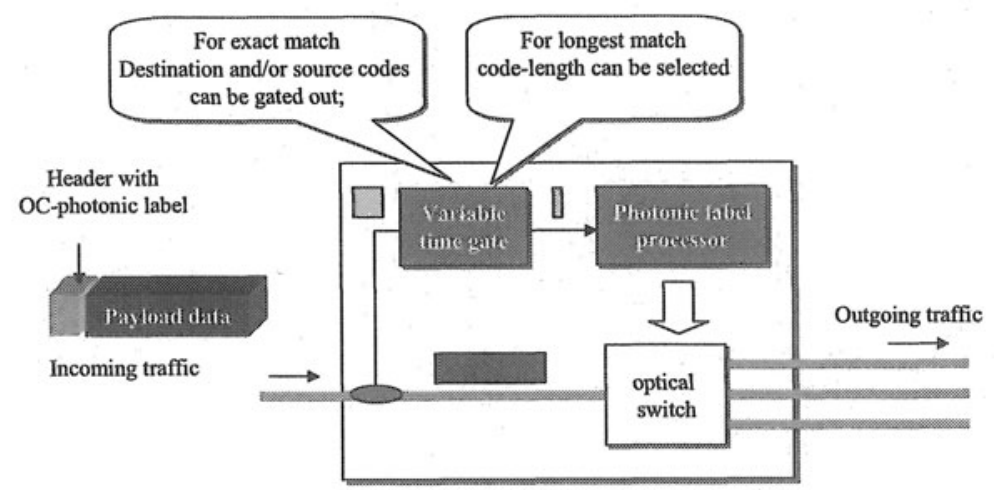

Figure 4. Architecture of $\mathrm{OC}$-photonic label processor for exact match and longest match 


\subsection{Flow classification}

The flow classification is an operation involved in Integrated Service (Intserve), which requires high speed packet processings [10]. Intserve is a service model which guarantees QoS on a per-flow basis. When the packets arrive, the router classifies the packets into a reserved flows and puts them on the appropriate queues to transmit when the outgoing link is ready. In the flow classification a router must examine every incoming packet and decide if the packet belongs to one of the reserved RVSP flows. An IP flow is identified by the five fields in the header. To determine if a packet matches an RSVP flow, the flow classification engine must compare the five fields of the incoming packet with the counterparts of all flows in the reservation table. If there is a match, the packet is allowed to be scheduled for the transmission. Flow classification must be performed on every packet, so it has to complete the lookup within the time budget for processing a single packet. For example, to support 64-byte packets at OC-192 $(10 \mathrm{~Gb} / \mathrm{s})$, the per-packet processing time is roughly $50 \mathrm{nsec}$, and then the time budget for each table lookup should be in a few nanoseconds regime. Therefore, performing flow identification at high speeds with a large number of flows is a challenging task.

The photonic flow classification using OC-photonic label is a powerful alternative to electronic approaches such as a direct memory lookup using a single memory access and binary search. The architecture of the photonic label processor for the flow classification as well as packet forwarding is shown in Fig.5. First, Gate switches divides the Fileds 1 and 2. Photonic processors 1 and 2 individually process Fields 1 and 2 , respectively, and the output signals from the processors generates the control signal to drive the $1 \times N$ optical switch to direct the packets to the appropriate output port. The photonic label can be processed based on the optical correlation between the codes. The optical correlation is performed by matched filtering in time domain. The optical gate switches select one of the two photonic labels in the header in front of the processors. 

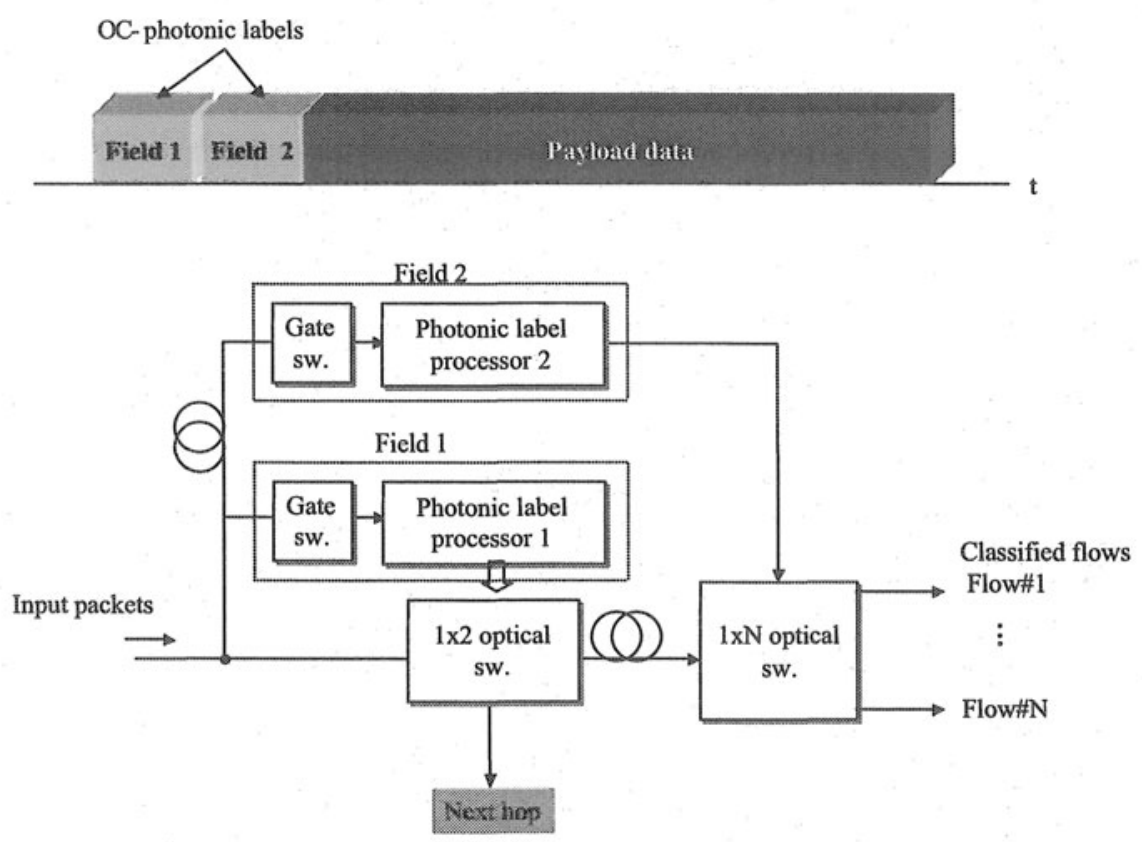

Figure 5. Architecture of photonic label processor for flow classification

\section{OPTICAL CODE-BASED PHTONIC LABEL}

\subsection{OC-photonic labels and their recognition based upon optical correlation}

The photonic label is simply a relatively short, fixed-length identifier that bears the information of either the LSP label, the IP address, flow label. Each label is mapped onto an optical code, a sequence of optical pulses, socalled chip pulses [11]. The chip itself is a short pulse, and the time duration of the sequence has to be within a bit time duration. The number of optical code increases as the code length increases, and it could be abundant in practical applications, contrast to the scarcity of wavelength resource for the labels. The photonic label recognition is based upon the optical correlation between the optical codes. The optical correlation is performed by matched filtering in time domain. For example, a family of optical codes of 4-chip long codes and their auto-correlation waveforms are illustrated in Fig.6. The code is bipolar, in which the phase of optical carrier of individual chip pulse 
takes two states of either 0 or $\pi$, representing binary value of 1 or -1 , respectively. As increasing the number of nodes, the longer code will be required. Unique to the label selector is that no optical logic operation is involved, and this is a key to the ultrahigh-speed operation of label processings.

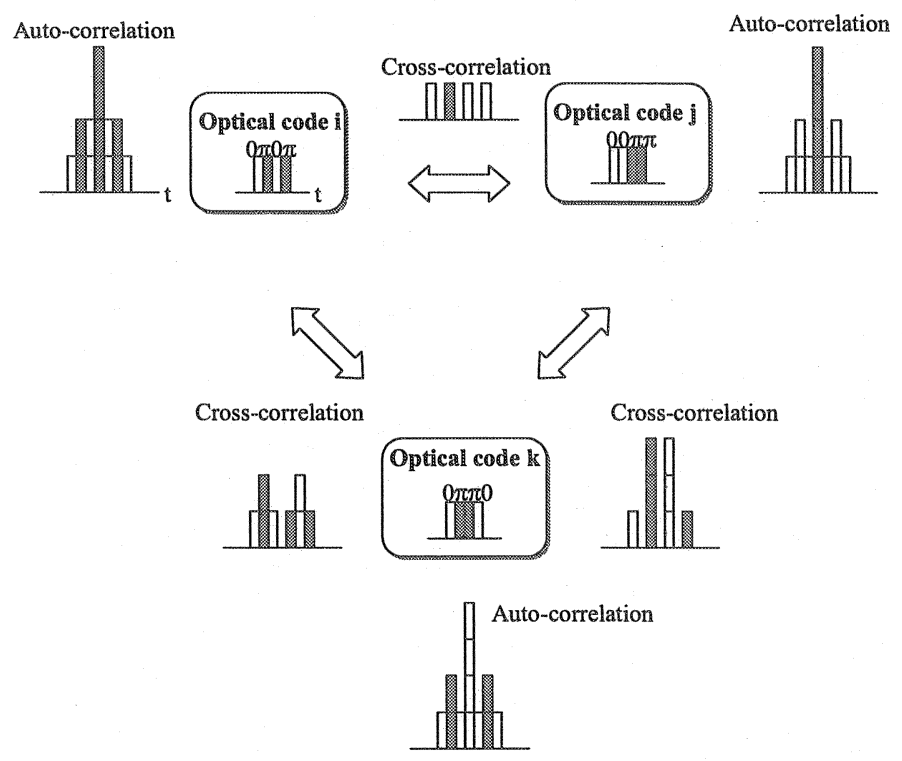

Figure 6. Optical correlation between two optical codes

\subsection{Optical encoder / correlator}

The optical encoder generates an OC-photonic label. Promising devices for the optical encoder are a waveguide type of tapped delay line [10] and a fiber Bragg grating (FBG) [12], shown in Figs.7 (a) and (b), respectively. The tapped delay line is a wave-guide device having an optical phase shifter on each arm, which is monolithically integrated on a silicon substrate. The FBG is a tiny optical fiber device, on which phase-shifted Bragg gratings are imprinted along the fiber axis. Both can generate bipolar optical codes. 
(a)

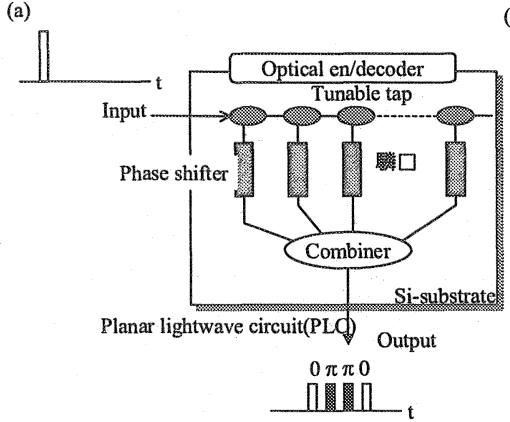

(b)

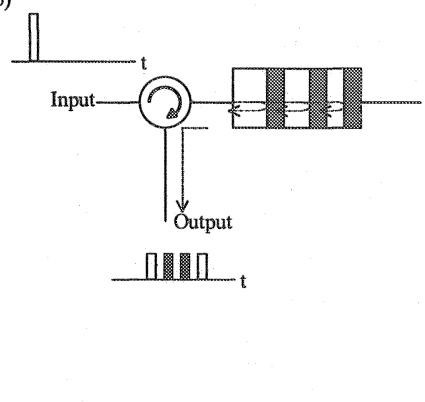

Figure 7. Optical encoder / correlator: (a) Waveguide tapped delay line and (b) FBG

The photonic label processor is structured with an optical correlator, a time-gate, optical thresholding, and an optical/electrical $(\mathrm{O} / \mathrm{E})$ conversion. The block diagram is schematically illustrated in Fig.8. Note that the identical optical device can be used for both the optical encoder and the optical correlator. The time gate, which follows the optical correlator, allows only the auto-correlation mainlobe to pass by, rejecting the sidelobes. The optical time gate has been achieved in an asynchronous system by using a semiconductor optical amplifier (SOA) based upon four-wave mixing (FWM)[13]. The optical gate is optically driven by the optical clock which is extracted from the incoming packet by using a mode-locked laser diode [14]. The gate width shorter than 10ps has been realized in the experiments $[13,14]$. Very recently, an ultrafast optical thresholding has been demonstrated with fiber-optic nonlinear loop mirror [15]. Several high-speed off-the-shelf optical space switches have now become available. 


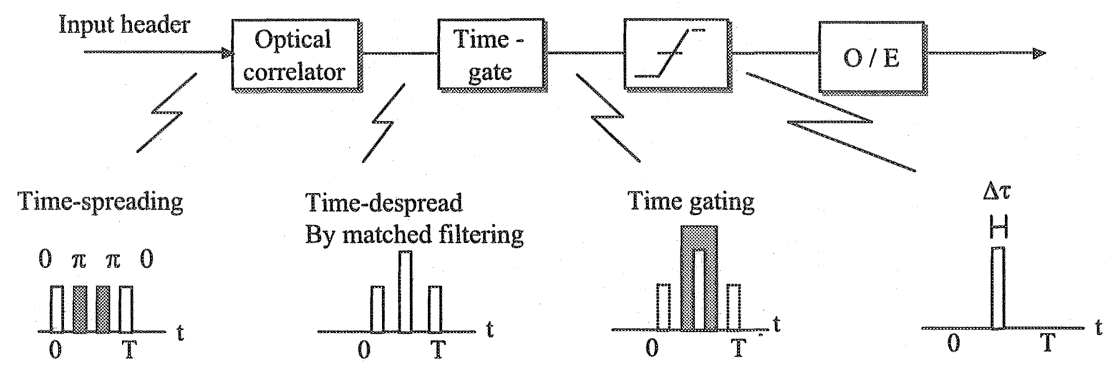

T: One bit time duration

Figure 8. Block diagram of photonic label processor

\section{EXPERIMENTS}

\subsection{Longest prefix match and exact match}

In Fig.10 the experimental setup of the longest prefix match is shown [9]. The programmable optical encoders/ decoders consist of tapped delay lines with thermo- controlled optical phase shifters. In variable optical gate a saturable absorber (SA) is used as the optical gate. The time window can be variable by pumping with a desired-length control optical code. An MLLD at $\lambda_{1}=1550 \mathrm{~nm}$ with a repetition rate of $10 \mathrm{GHz}$ is used as the light source. The optical encoder generates an 8-chip binary phase shift keying (BPSK) optical code with a chip time interval of $5 \mathrm{ps}$. The optical decoders generate correlation outputs. The peaks of the auto-correlation peaks are compared by using a dual-pin PD. 


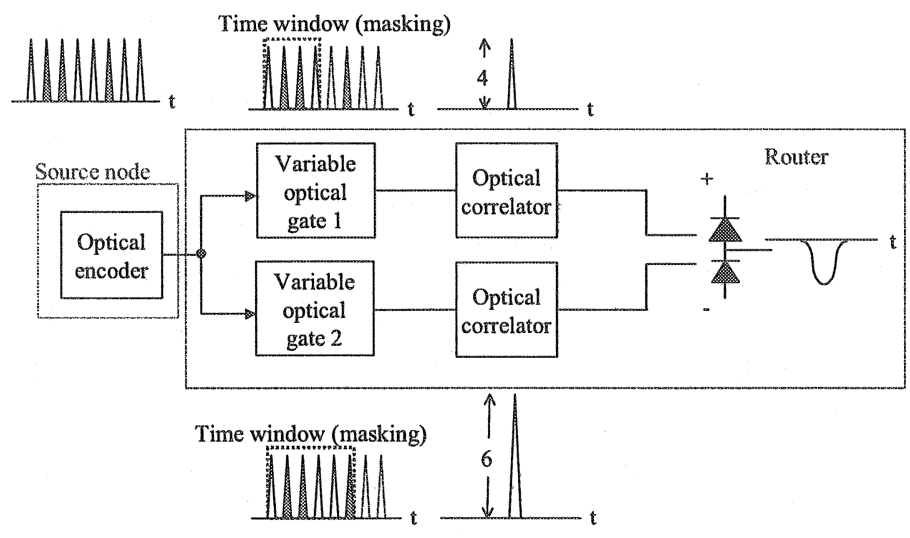

Figure 9. Experimental setup for longest prefix match

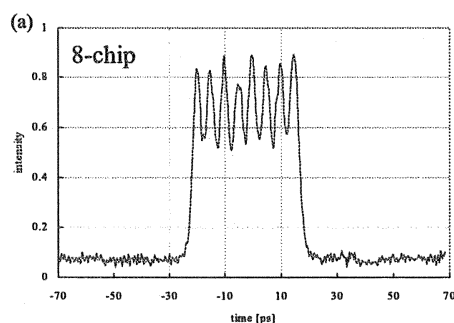

(b)

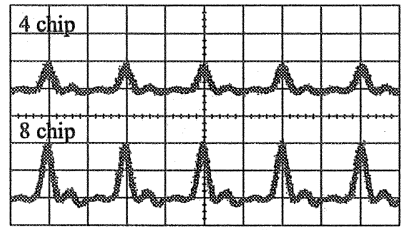

$50 \mathrm{ps} / \mathrm{div}$

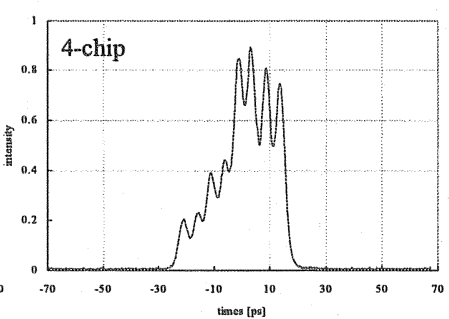

(c)

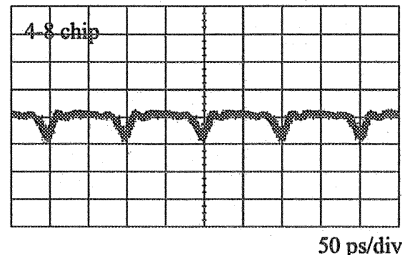

Figure 10. (a) Optically-gated control codes, (b) optical decoder outputs: auto-correlation waveforms, and (c) output of a dual-pin PD

The optical code at $10 \mathrm{~Gb} / \mathrm{s}$ is duplicated and launched into variable optical gates. Time windows of optical gates are set to be 4- and 8-chip long. The same 4- and 8-chip codes are assigned to optical decoders 1 and 2, 
respectively, and thus the decoder outputs will show the auto-correlation functions of optical codes. The optically-gated control optical codes, the decoder outputs before and after the dual-pin PD are shown in Figs.10 (a), (b), and (c), respectively. The optical signal levels exactly correspond to the autocorrelation peaks, 4 and 8 in Fig.10 (b). The subtraction of 4 minus 8 in electrical domain shows the photocurrent of minus sign in Fig. 10 (c), and this allows us to determine that 8-chip code wins the longest matching.

\subsection{Photonic flow classification}

The experimental setup for photonic flow classification is shown in Fig.11. In this experiment only the merge and split of packets are performed, and the packet forwarding is omitted for simplicity. It consists of an optical packet transmitter, a photonic label processor, and an $1 \times 2$ optical switch. The optical packet transmitter consists of a $1.3 \mathrm{ps}-10 \mathrm{GHz}-\mathrm{MLLD}, \mathrm{LiNbO}_{3}$ intensity modulators (LN-IMs), and two optical encoders. At the receiver side, photonic label processor is composed of an optical gate, an optical decoder, a photodiode, and a gate signal generator. The optical encoder/decoder is a programmable tapped delay-lines with thermocontrolled optical phase shifters.

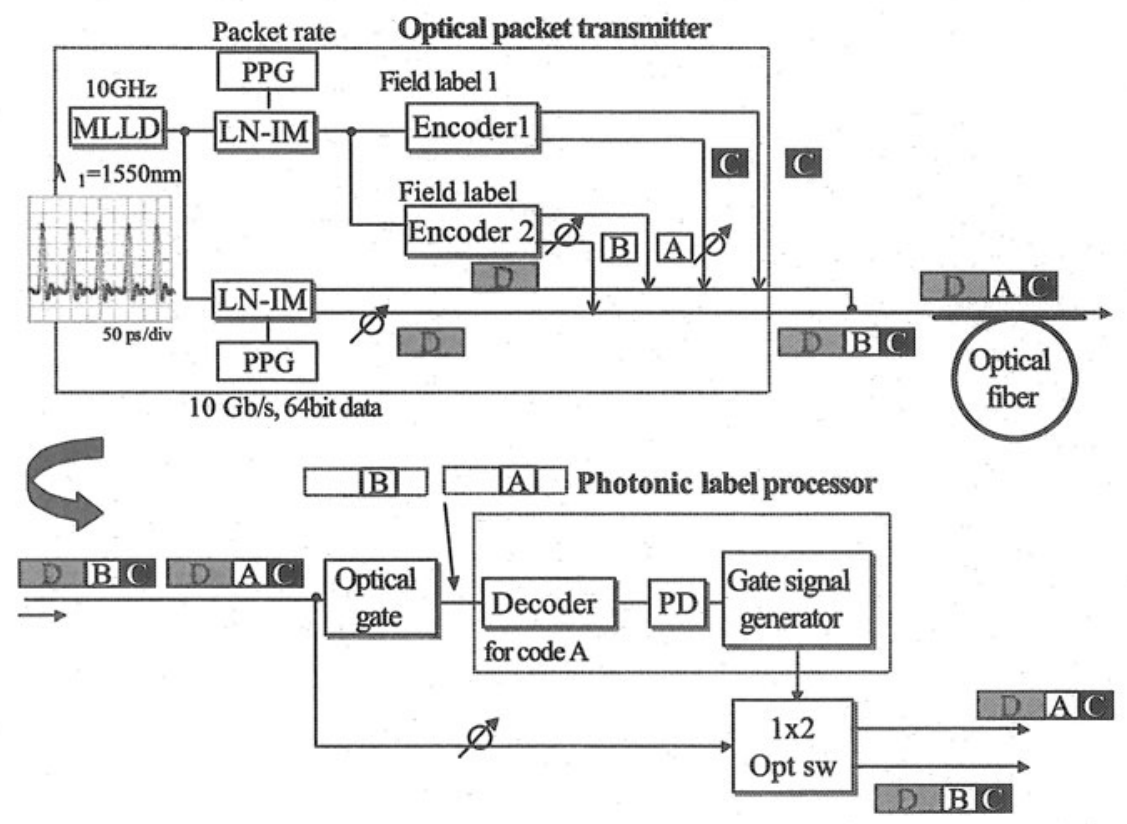

Figure 11. Experimental setup for photonic flow classification 
The photonic label processor performs optical correlation between the optical code of arriving packet and the assigned code at the receiver. If the codes match, the auto-correlation output is obtained, and after the photodetection it generates the gate signal. The gate signal drives the $1 \times 2$ optical switch to direct the packet to the upper output port. Otherwise, the cross-correlation output is obtained, but no gate signal is generated to hold the optical switch in the original state, and the packet emerges from the lower output port.

The experiment of the photonic flow classification is conducted for packets at the data bit rate of $10 \mathrm{Gbit} / \mathrm{s}$. The measurements are made before and after 50km-long SMF+RDF fiber transmission. The measured waveforms of two generated 64-bit long packets with a pair of photonic labels of different sets of Field labels 1 and 2 and Field labels 1' and 2' in the header, generated control signals, and the input and classified packets are shown in Fig.12. It is confirmed that the optical switch, controlled by photonic label processor, classifies the packets to the two different output ports according to the label entries in the reservation talble in the processor. The BERs less than $10^{-9}$ were obtained both before and after the transmission, thus guaranteeing the proper operation. The power penalties are presumably due to the ASE noise of the EDFAs used in the transmission.

\section{CONCLUSION}

It has been shown that OC-MPLS is a framework in which circuitswitched routing as well as optical packet switching can be supported. We will focus on the novel applications of OC-MPLS to demonstrate its versatile capability. The application such as LSP switching, IP packet routing, and flow classification have been investigated. In the optical implementations, two types of match algorithms of OC-photonic labels such as exact match for the LSP and flow classification and longest prefix match for IP packet routing have been presented. Finally, the exact match, longest prefix match, and photonic flow classification have been experimentally demonstrated at the bit rate of $10 \mathrm{~Gb} / \mathrm{s}$.

\section{REFERENCES}

[1] N. Ghani, "On IP-over-WDM Integration," IEEE Communications Magazine, pp.72-84, March 2000D. 
[2] O. Awduche, Y. Rekhter, J. Drake, and R. Coltun, "Multi-Protocol Lambda Switching: Combining MPLS Traffic Engineering Control with Optical Crossconnects," IETF Internet Draft, draft-awduche-mpls-te-optical-02.txt.

[3] U. Black, MPLS and Label Switching Networks, Prentice Hall Inc., 2001.

[4] M. Murata and K. Kitayama, "A perspective on photonic multiprotocol label switching," IEEE Network Magazine., vol.15, pp.56-63, July/Aug. 2001.

[5] K. Sato and K. Kitayama, "Cutting-edge technologies on photonic backbone networks Envisaged IP over photonic networks of the year of 2010(2)-," to appear in the July issue of IEICE.

[6] A. Carena, M.D. Vaughn, R. Gaudino, M. Shell, and D.J. Blumenthal, "OPERA: An Optical Packet Experimental Routing Architecture with Label Switching Capacity," Journal on Lightwave Technology, Vol.16, pp.12135-2145, 1998.

[7] D. K. Hunter, et al., "WAPSNET: A wavelength switched packet network," IEEE Communications Magazine, pp.120-129, March 1999.

[8] B. Davie, P. Doolan. And Y. Rekhter, Switching in IP Networks, Morgan Kaufmann Piblishers, Inc, 1998.

[9] M. Onohara, N. Wada, W. Chujo, and K. Kitayama, "Optical code-based IP longestprefix-match using variable-length optical gate," Optoelectronics and Communications Conference (OECC2001), TuJ2 (Sydney, July 2001).

[10] Z. Wang, Internet QoS, Morgan Kaufmann Piblishers, Inc, 2001.

[11] K. Kitayama, N. Wada, and H. Sotobayashi, "Architectural considerations of photonic IP router based upon optical code correlation (Invited)," IEEE J. Lightwave Technol., vol.18, pp.183-1844, 2000.

[12] M. C. Cardakli, D. Gurkan, S. A. Havstad, A. E. Willner, "Variable-bit-rate Header Recognition for Reconfigurable Networks using Tunable Fiber-Bragg-Gratings as Optical Correlators," 2000 Optical Fiber Conference (OFC2000), TuN2, Baltimore, March 2000.

[13] N. Wada and K. Kitayama, "Photonic IP Routing using Optical Codes: 10Gbit/s Optical Packet Transfer Experiment," 2000 Optical Fiber Conference (OFC 2000), WM51, Baltimore, March 2000.

[14] K. Kitayama, H. Sotobayashi, and N. Wada, "Optical Code Division Multiplexing (OCDM) and its Applications to Photonic Networks," IEICE Transactions on Fundamentals, Vol. E82-A, pp. 2616-2626, 1999.

[15] H. Sotobayashi, W. Chujo, and K. Kitayama, "1.6 bit/s/Hz, 6.4 Tbit/s OCDM/WDM (4 OCDM x $40 \mathrm{WDM}$ x $40 \mathrm{Gbit} / \mathrm{s})$ transmission," 27th European Conference on Optical Communications (ECOC2001), PD.M.1.3 (Amsterdam 2001). 


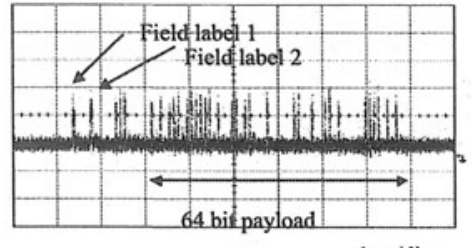

$1 \mathrm{~ns} / \mathrm{div}$

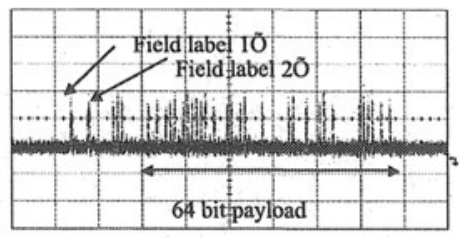

$1 \mathrm{~ns} / \mathrm{div}$

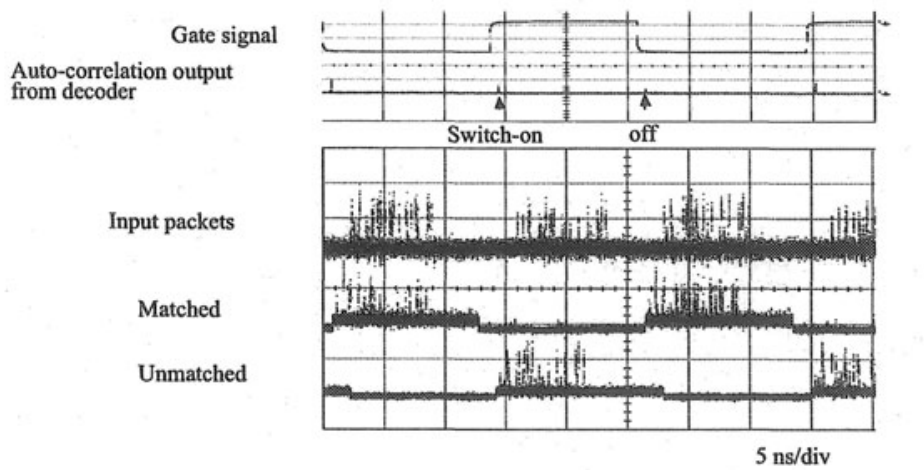

Figure 12. Measured waveforms; generated 64-bit long packets with two photonic labels in the header (on the top), generated control signals (in the middle), and the merged and classified packets (on the bottom) 